\title{
Loss compensation in metal-dielectric layered metamaterials
}

\author{
Roman S. Savelev, ${ }^{1}$ Ilya V. Shadrivov, ${ }^{2}$ Pavel A. Belov, ${ }^{1}$ Nikolay N. Rosanov, ${ }^{1,3}$ Sergey V. Fedorov, ${ }^{1,3}$ \\ Andrey A. Sukhorukov, ${ }^{2}$ and Yuri S. Kivshar ${ }^{1,2}$ \\ ${ }^{1}$ National Research University of Information Technologies, Mechanics and Optics (ITMO), St. Petersburg 197101, Russia \\ ${ }^{2}$ Nonlinear Physics Centre, Research School of Physics and Engineering, Australian National University, Canberra ACT 0200, Australia \\ ${ }^{3}$ Vavilov State Optical Institute, Birzhevaya liniya 12, St. Petersburg 199034, Russia
}

(Received 20 December 2012; revised manuscript received 3 March 2013; published 27 March 2013; publisher error corrected 29 March 2013 )

\begin{abstract}
We analyze a possibility of gain-induced compensation of losses in a one-dimensional layered structure, consisting of alternating layers of metal and dielectric with gain. Using an exact approach without effective medium approximations, we demonstrate that losses can be completely compensated for a certain value of the longitudinal component of the wave vector and partially reduced for other wave numbers. In the systems with larger values of the gain coefficient, convective instabilities can appear. At certain frequencies, losses can be greatly reduced for a broad range of the incidence angles. We find that in the canalization regime when the isofrequency contours are close to straight lines, the loss compensation becomes possible for a wide spatial spectrum being useful for improvement of the subwavelength imaging capability.
\end{abstract}

DOI: 10.1103/PhysRevB.87.115139

PACS number(s): 78.67.Pt, 42.25.-p

\section{INTRODUCTION}

In recent years, we observed a growing interest in the study of the physics of metal-dielectric layered metamaterials (MDLMs), which can exhibit effective hyperbolic dispersion. ${ }^{1}$ Applications of such MDLMs include superlenses with subwavelength resolution, ${ }^{2-4}$ hyperlenses, ${ }^{5}$ electromagnetic cloaking, ${ }^{6}$ nanolithography, ${ }^{7}$ and others. However, the use of such structures at optical frequencies is significantly limited by the effect of losses which originate from the constituent metal layers. One of the most promising ways to mitigate the losses is to incorporate a gain medium, such as organic dye molecules or semiconductor quantum wells or quantum dots, in dielectric layers. ${ }^{8-10}$ Such an approach was suggested theoretically and applied experimentally to various systems such as singleperiodic and double-periodic photonic crystals, ${ }^{11,12}$ fishnet metamaterials with negative refractive index, ${ }^{13-15}$ structures supporting localized surface plasmon polaritons, ${ }^{16-19}$ multilayered metal-dielectric metamaterials, ${ }^{20-22}$ and others. ${ }^{23-28}$

One of the interesting features of MDLM, schematically shown in Fig. 1, is that negative metal permittivity leads to the appearance of the hyperbolic isofrequency surface in the longwavelength limit, when the effective medium approximation can be applicable. Such a dispersion is associated with a strong modification of the spontaneous emission rate..$^{29,30} \mathrm{We}$ note that an exact description of an optical response shows that such structures can demonstrate strong nonlocal effects due to the excitation of surface plasmon polaritons (SPP), and often the effective medium approach cannot provide an accurate description of the system even in the long-wavelength limit. ${ }^{31-33}$ Hyperbolic-like dispersion relation for eigenmodes of such structures can be obtained only for relatively low frequencies, and it depends strongly on the ratio of the layer thicknesses. ${ }^{33}$ Loss compensation for such structures was claimed to be achieved in the "hyperbolic" regime for the longitudinal propagation; ${ }^{22}$ however, loss compensation for a range of incident angles remained an open problem. It was shown ${ }^{33}$ that at higher frequencies (but still when the wavelength of the incident radiation is much larger than the period of the structure), two branches with hyperbolic and elliptical dispersions can coexist, which is in a sharp contrast to the prediction of effective medium theory where only one type of the modes can exist for a given frequency. This regime could be interesting, e.g., in the case of pulse propagation, due to a strong dependence of optical response of such a structure on frequency. Another interesting case is the so-called canalization regime, ${ }^{3}$ when for a certain range of longitudinal wave vector components the isofrequency contours form almost straight lines. In this case, the field distribution is transmitted across the layers from one side to another, and it is useful to estimate the effect of losses in such systems and a possible improvement of its performance.

In this paper, we study different regimes of the loss compensation in MDLM structures, and demonstrate that using a gain medium it is possible to compensate loss effects in the cases of the transverse and longitudinal wave propagation.

The paper is organized as follows. In Sec. II, we formulate our model of MDLMs with a gain in the dielectric layers. In Sec. III, we discuss our numerical results. Section IV concludes the paper and outlines further perspectives.

\section{MODEL AND EQUATIONS}

We study the propagation of a TM-polarized plane wave with the angular frequency $\omega$ in a MDLM consisting of alternating layers of metal (silver) and dielectric with a gain (see Fig. 1). In our calculations, we consider the parameters of an organic dye mixed in epoxy, which is a frequently used gain material in plasmonic experiments. ${ }^{8,16,17}$ For near-infrared frequencies, one can also use semiconductor quantum dots, which can provide larger gain coefficients and they are also compatible with semiconductor manufacturing technologies. ${ }^{14}$ For the complex permittivity of silver, we use the Drude formula:

$$
\varepsilon_{1}(\omega)=1-\frac{\omega_{p}^{2}}{\omega^{2}+i \gamma \omega},
$$

where $\omega_{p}=2 \pi c / \lambda_{p}$ is the plasma frequency, $\lambda_{p}=250 \mathrm{~nm}$, $c$ is the speed of light, and $\gamma=2 \pi \times 10^{13} \mathrm{~s}^{-1}$ is the damping rate. Note that the value of the damping rate $\gamma$ in a thin metal 


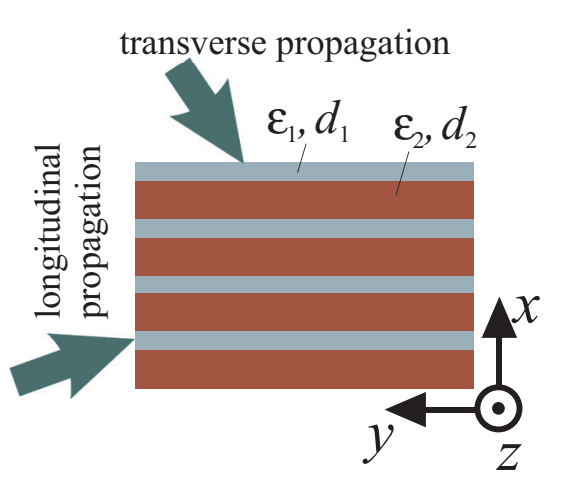

FIG. 1. (Color online) Geometry of the MDLM structure, consisting of the alternating metal and dielectric layers (subscripts 1 and 2 , respectively).

layer is greater than that in a bulk metal and it depends on the thickness of the layer and quality of the silver film. ${ }^{34}$ That is why we use a larger value of damping than it is measured for a bulk metal. ${ }^{35}$ Dielectric permittivity of the active dielectric is taken in the form $\varepsilon_{2}(\omega)=\varepsilon_{2}^{\prime}(\omega)-i \varepsilon_{2}^{\prime \prime}(\omega)$. Here $\varepsilon_{2}^{\prime}=2.72$ is the real part of dielectric permittivity, dominated by the permittivity of the epoxy and therefore it is almost constant. ${ }^{22}$ Imaginary part $\varepsilon_{2}^{\prime \prime}$ is negative, and its frequency dependence for most gain media can be approximated with Lorentzian line shape. Since we consider the propagation of a continuous plane wave in the linear regime, we assume that at a certain frequency $\varepsilon_{2}^{\prime \prime}$ is constant, because we calculate the value of gain required for the full compensation of losses at a certain frequency in some particular direction. In experiments the maximum value of $\varepsilon_{2}^{\prime \prime}$ does not exceed approximately 0.04 for most dyes, ${ }^{17,22}$ and $0.1-0.3$ for semiconductor gain. ${ }^{36}$

We use the dispersion equation for eigenmodes of an infinite one-dimensional layered structure: ${ }^{37}$

$$
\begin{aligned}
\cos \left(k_{x} D\right)= & \cos \left(q_{1} d_{1}\right) \cos \left(q_{2} d_{2}\right) \\
& -\frac{1}{2}\left(\frac{\varepsilon_{2} q_{1}}{\varepsilon_{1} q_{2}}+\frac{\varepsilon_{1} q_{2}}{\varepsilon_{2} q_{1}}\right) \sin \left(q_{1} d_{1}\right) \sin \left(q_{2} d_{2}\right),
\end{aligned}
$$

where $b_{i}=q_{i}$ for TE polarization and $b_{i}=q_{i} / \varepsilon_{i}$ for TM polarization, $q_{i}=k_{0} \sqrt{\varepsilon_{i}-\left(k_{y} / k_{0}\right)^{2}}$ are the normalized $x$ components of wave vectors, $i=\{1,2\}, k_{x}$ is the $x$ component of the Bloch wave vector, $d_{1}=D / 3, d_{2}=2 D / 3$ are the thicknesses of metal and dielectric layers, respectively, $D=60 \mathrm{~nm}$ is the structure period, and $k_{0}=\omega / c$.

Two main regimes are considered here. First is the transverse propagation, when the longitudinal component of wave vector $k_{y}$ is real valued and $k_{x}$ can be complex valued. Second is the longitudinal propagation (end-fire coupling regime), when $k_{x}$ is real valued and $k_{y}$ is complex valued.

For transverse propagation, the isofrequency contours have two symmetric branches in the first Brillouin zone. To determine which branch corresponds to which direction of energy propagation, we calculate the $x$ component of the Poynting vector:

$$
S_{x}=\frac{1}{2} \operatorname{Re}\left(E_{y} H_{z}^{*}\right) .
$$

Depending on the mutual direction of $S_{x}$ and $k_{x}$, waves can be either forward or backward. As we show in Fig. 2(a), the

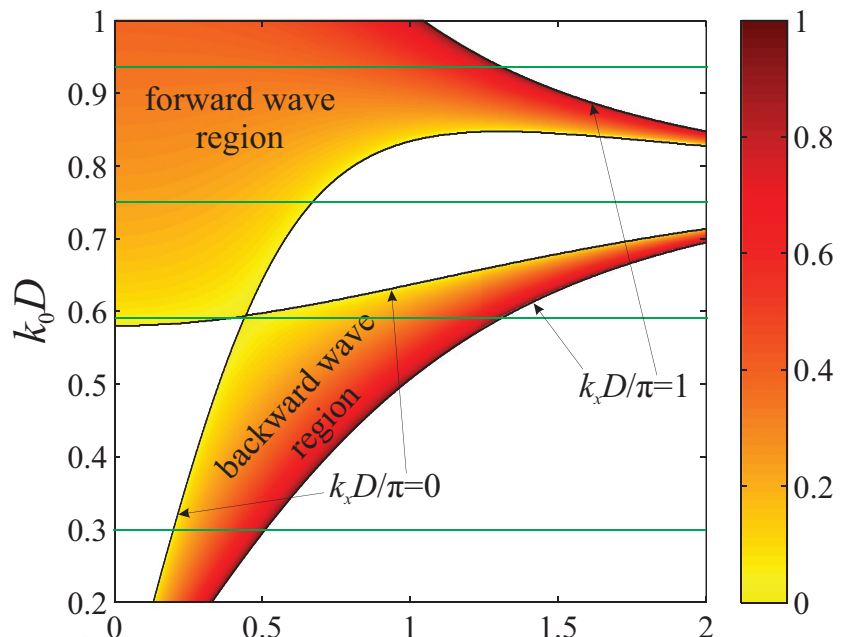

(a)
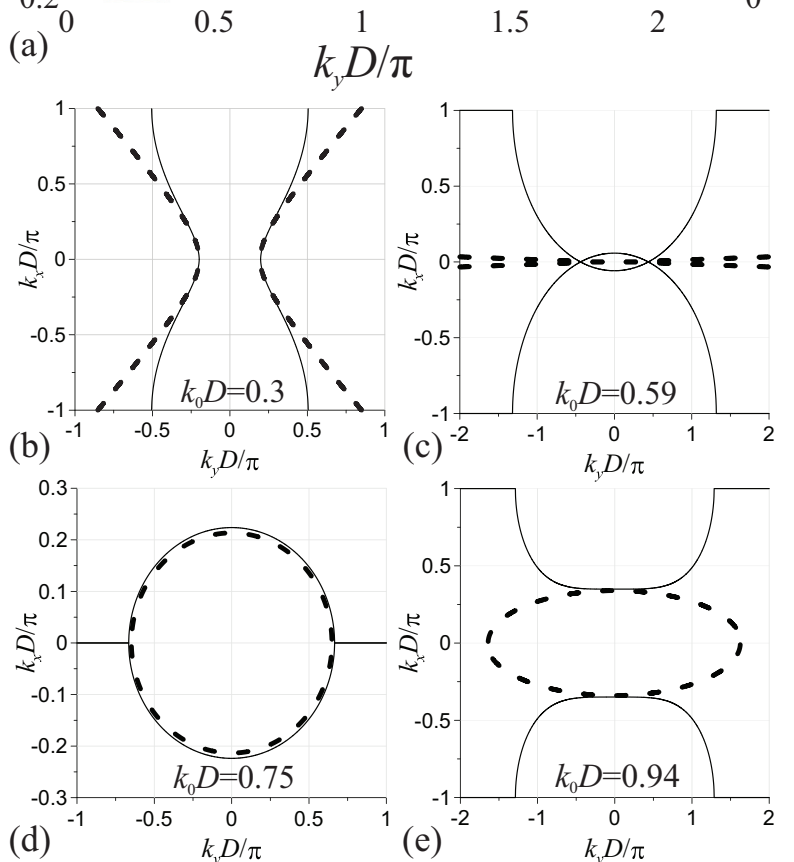

FIG. 2. (Color online) (a) Dispersion diagram; different values of the normalized transverse component of Bloch wave vector $k_{x} D / \pi$ are shown in different colors. Horizontal lines correspond to the frequencies used in (b)-(e). Isofrequency contours in the case of zero losses and gain for (b) $k_{0} D=0.3$, (c) $k_{0} D=0.59$, (d) $k_{0} D=0.75$, and (e) $k_{0} D=0.94$; solid lines are solutions of Eq. (1); dashed lines correspond to the effective medium approximation.

modes corresponding to hyperbolic contours are backward, while elliptic modes are forward.

For the case of no losses or gain, when $\gamma=0$ and $\varepsilon_{2}^{\prime \prime}=0$, the dispersion diagram is shown in Fig. 2(a). Color shows the value of the normalized $x$ component of the Bloch wave vector $k_{x} D / \pi$. In the regime of transverse propagation, we consider three representative cases. In the first case, $k_{0} D=0.3$, the isofrequency contour is similar to hyperbolic [Fig. 2(b)]. In this low frequency range MDLM can be considered as a hyperbolic metamaterial. In the second case, $k_{0} D \approx 0.58-0.85$, there are two types of isofrequency curves-elliptic and hyperbolic. As will be shown later, gain required for a complete compensation almost does not depend on $k_{y}$ for $k_{0} D=0.59$ and $k_{0} D=0.75$, 
so these two frequencies will be of interest in this case. Figures 2(c) and 2(d) show isofrequency contours for these values of $k_{0} D$. The third case, $k_{0} D=0.94$, corresponds to a canalization regime ${ }^{3}$ [Fig. 2(e)]. In the case of the longitudinal propagation the canalization regime does not have interesting features, so only two first cases will be considered. For more detailed information about isofrequency contours for such structures, see Refs. 32 and 38.

Dashed curves in Figs. 2(b)-2(e) correspond to the effective medium approach with the dispersion equation

$$
\frac{k_{x}^{2}}{\varepsilon_{\perp}}+\frac{k_{y}^{2}}{\varepsilon_{\|}}=k_{0}^{2},
$$

where

$$
\varepsilon_{\|}=\frac{\varepsilon_{1} d_{1}+\varepsilon_{2} d_{2}}{D}, \quad \varepsilon_{\perp}=\frac{\varepsilon_{1} \varepsilon_{2} D}{\varepsilon_{2} d_{1}+\varepsilon_{1} d_{2}} .
$$

Effective medium approach works well only when $k_{0} D$ is much less than "epsilon-near-zero" (ENZ) frequency, where $\operatorname{Re}\left(\varepsilon_{\|}\right)$ vanishes, and it can predict only one type of dispersion-either hyperbolic or elliptical. ${ }^{38}$

We note that the structure with gain layers which are infinite in the transverse direction can become unstable. There one can observe two types of instabilities, convective and absolute. ${ }^{39}$ Absolute instability corresponds to the exponential growth of the electromagnetic energy in time, in a certain spatial region. This effect corresponds to the modes with a complex-valued frequency $\omega$ with $\operatorname{Im}(\omega)<0$. Absolute instability can be neglected in our case due to the very high threshold gain. It was shown earlier ${ }^{12}$ that for dielectric photonic crystals absolute instability or the laser generation threshold has to be taken into account only for high frequencies $\left(k_{0} D \gtrsim 1\right)$. The presence of metal in our structure increases the threshold of laser generation dramatically. It is also worth mentioning that more realistic cases of reflection from semi-infinite or finite-sized uniform or periodic amplifying layers require a more complex approach to determine a lasing threshold. ${ }^{40,41}$

Convective instability or amplified spontaneous emission corresponds to the existence of modes with real-valued frequency and complex-valued longitudinal wave vector component $k_{y}$. When $\operatorname{Im}\left(k_{y}\right)<0$ for the propagation in positive $y$ direction or $\operatorname{Im}\left(k_{y}\right)>0$ otherwise, modes are amplified along the propagation direction, which means that any spontaneous emission in the system can become a source of the noise, that will be amplified, returning dye molecules from excited to the ground state, and providing a mixing of a noise radiation with the main signal. It is well known that this effect has a detrimental influence on lasers. ${ }^{42}$ In metamaterials, amplified spontaneous emission has been observed at the interface between a thin gold film and a resonant gain medium in the form of the polymer film, doped with $\mathrm{PbS}$ quantum dots. ${ }^{43}$ Despite a high pump intensity, increase in SPP propagation length was limited to only $30 \%$ due to amplified spontaneous emission. It means that in the linear regime one should avoid the existence of such amplified modes, both TE and TM polarized. Here we will not analyze the system dynamics in unstable regimes when one should employ a more detailed and realistic model, which should consider finite structure and take into account nonlinear effects of gain saturation that limit the signal growth, as well as quantum electrodynamics effects.

\section{RESULTS AND DISCUSSIONS}

\section{A. Transverse propagation}

The transverse propagation regime describes the modes, which would be excited in the structure, if it was finite in the $x$ direction. In such a case, $k_{y}$ is real and $k_{x}$ is determined as a complex-valued inverse cosine, according to Eq. (1).

Color maps in Figs. 3(a) and 3(b) show a value of gain required for the complete compensation of losses as a function of $k_{0} D$ and $k_{y} D / \pi$ for TM- and TE-polarized modes, respectively. For an infinite structure, the conditions of the full compensation and conditions of amplified spontaneous emission threshold are the same, so the TE polarization has to be considered in order to avoid the appearance of the TE-polarized amplified modes.

Since for the modes propagating in the positive $x$ direction the negative imaginary part of $k_{x}$ corresponds to the unstable
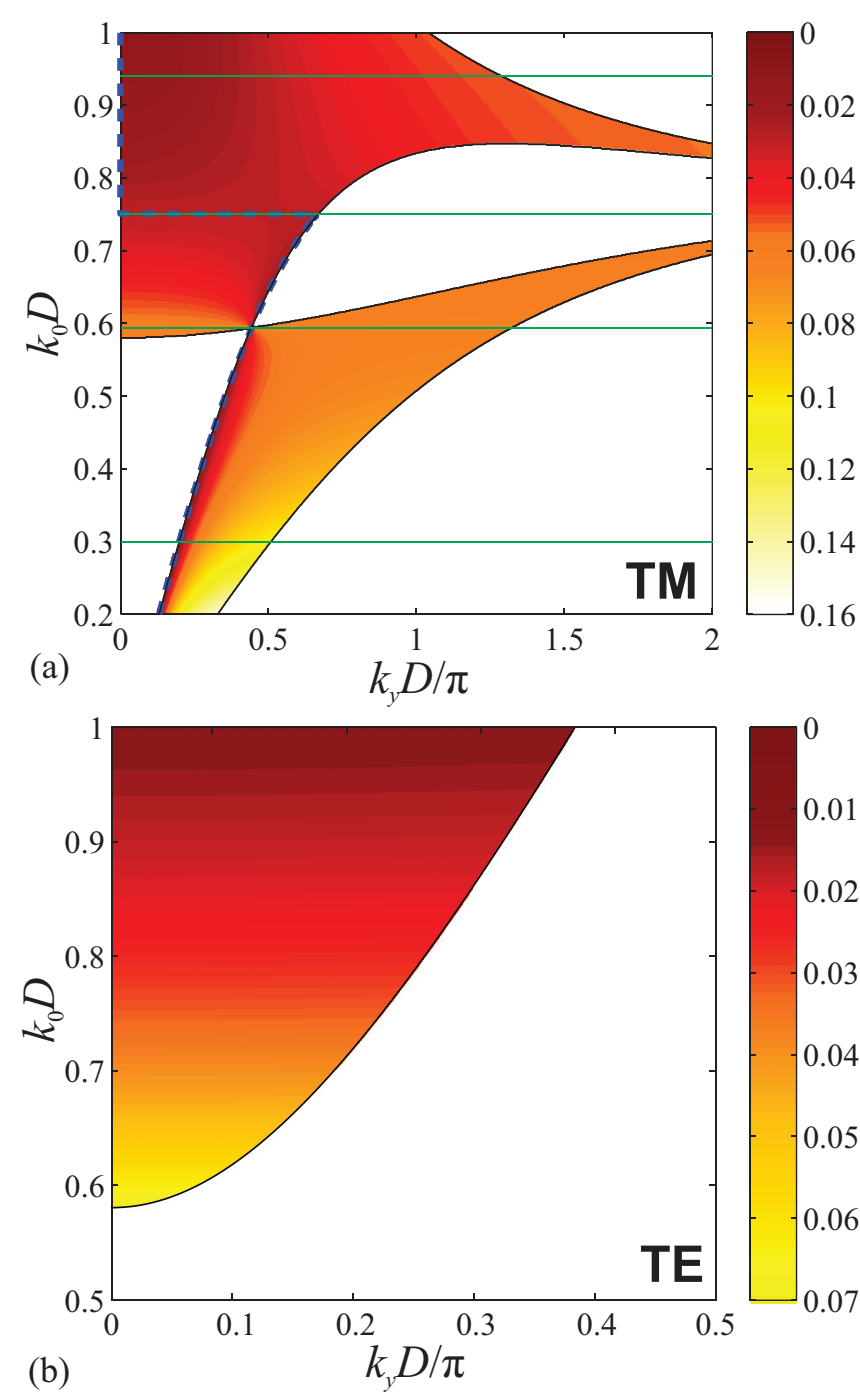

FIG. 3. (Color online) Dependencies of gain required for the full loss compensation $\varepsilon_{2}^{\prime \prime}$ on the normalized frequency $k_{0} D$ and the longitudinal component of the wave vector $k_{y} D / \pi$ for (a) TM polarization and (b) TE polarization. Thick dashed line in (a) shows $k_{y} D / \pi$, where $\varepsilon_{2}^{\prime \prime}$ has a minimum value. Horizontal lines in (a) indicate frequencies considered in Fig. 4. 

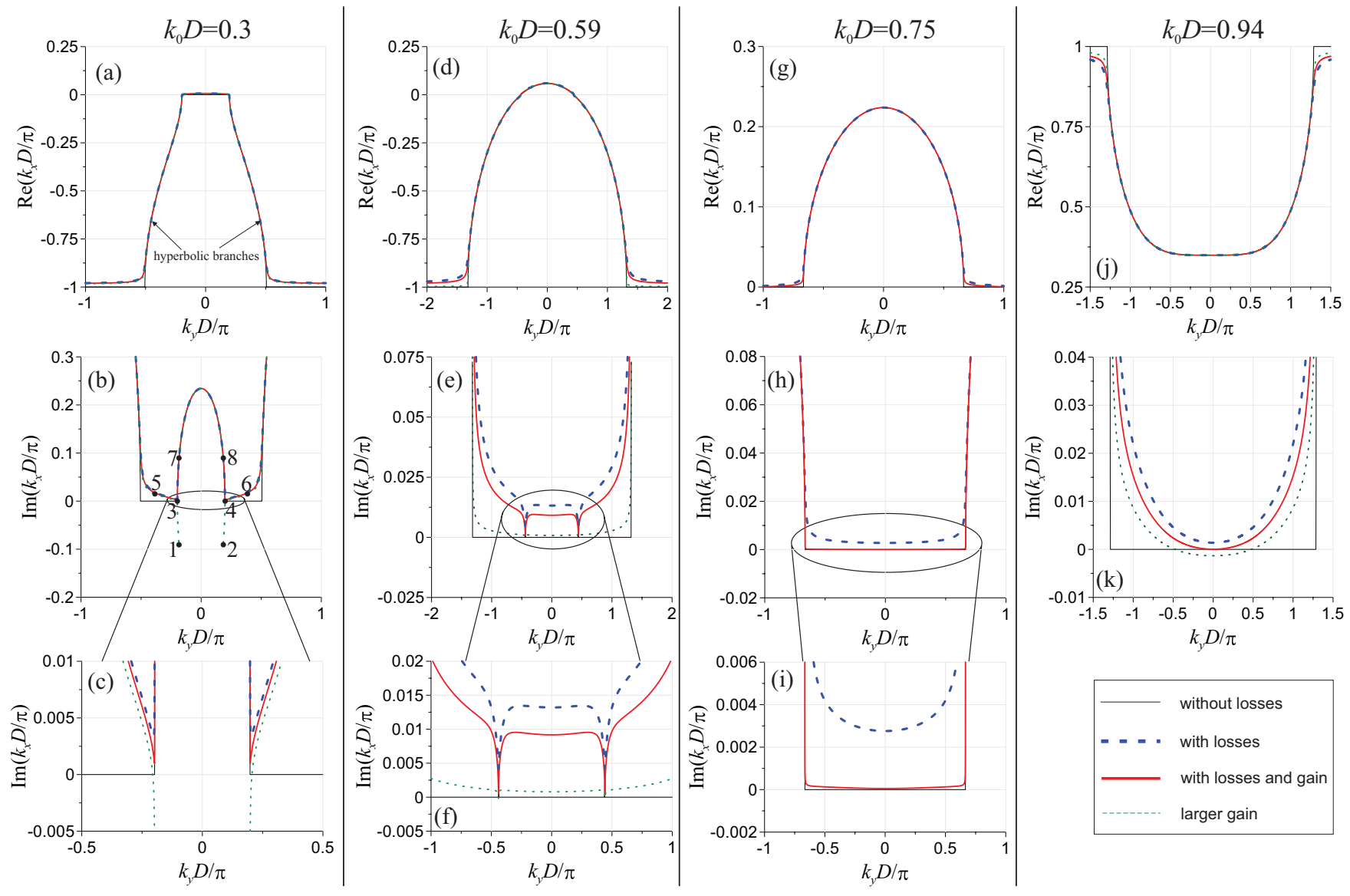

FIG. 4. (Color online) Examples of the isofrequency contours $k_{x}\left(k_{y}\right)$ for the transversely propagating modes (complex-valued $k_{x}$ and real-valued $k_{y}$ ) for different frequencies: (a)-(c) $k_{0} D=0.3$, (d)-(f) $k_{0} D=0.59$, (g)-(i) $k_{0} D=0.75$, and (j)-(k) $k_{0} D=0.94$. Figures (c), (f), and (i) show the zoomed-in regions from (b), (e), and (h), respectively. In (b) $1 \rightarrow 7$ and $2 \rightarrow 8$ are the points, where $\operatorname{Im}\left(k_{x}\right)$ has discontinuities and changes its sign; points 1-6 correspond to respective curves in Fig. 5. Thin solid curves correspond to the case of zero losses, dashed curves to the case with losses, and thick solid and short-dashed curves to the case with both losses and gain.

state, for a certain value of $k_{0} D$ we have to limit the value of gain: $\varepsilon_{2}^{\prime \prime} \lesssim \varepsilon_{2 \min }^{\prime \prime}$, where $\varepsilon_{2 \min }^{\prime \prime}$ is a minimal value of the critical gain for both polarizations and all directions of propagation for a given frequency $k_{0} D$. For TM-polarized modes the minimal value of $\varepsilon_{2}^{\prime \prime}$ is shown in Fig. 3(a) with a thick dashed line. For $k_{y}=0$ there is no difference in TE- and TM-polarized modes, so the threshold values of gain are the same. We find that the value of the critical gain for TE-polarized modes almost does not depend on $k_{y}$, so it is always greater than or equal to the minimal value of gain for TM-polarized modes. Therefore, the value of $\varepsilon_{2 \min }^{\prime \prime}$ is determined only by TM polarization, and TEpolarized amplified spontaneous emission can be neglected.

We reveal the important feature that at $k_{0} D=0.59$ (which is close to ENZ frequency) and $k_{0} D=0.75$ (close to SPP resonance frequency) in the transverse propagation regime, the critical value of gain has a very weak dependence on $k_{y}$.

All the considered frequencies are shown in Fig. 3(a) with the horizontal lines. The isofrequency contours for these frequencies are shown in Fig. 4. We see that small values of $\varepsilon_{1,2}^{\prime \prime}$ have a weak influence on $\operatorname{Re}\left(k_{x}\right)$, so the real parts with and without gain almost coincide. We prove this using perturbation theory (see the Appendix).
At low frequencies $\left(k_{0} D=0.3\right)$ there are only hyperbolic modes in the structure, and we see in Figs. 4(a)-4(c) that for $\varepsilon_{2}^{\prime \prime}=0.008$ (thick solid line), $\operatorname{Im}\left(k_{x} D / \pi\right)$ has minima at two symmetric $k_{y}$ values. For the modes propagating in the positive $x$ direction, determined according to the calculated Poynting vector, with an increase of gain to $\varepsilon_{2}^{\prime \prime}=0.02$, the isofrequency contours split into two parts [short-dashed curves in Figs. 4(b) and 4(c)] with jump discontinuities $1 \rightarrow 7,2 \rightarrow 8$. The field distribution $\left|H_{z}(x)\right|^{2}$ and $x$ component of the Poynting vector are shown in Fig. 5. For $k_{y} \approx \pm 0.4$ [points 5 and 6 in Fig. 4(b)], the average value of $S_{x}$ decreases with distance [Figs. 5(a) and 5(b)]. For $k_{y} \approx \pm 0.2$ [points 3 and 4 in Fig. 4(b)], $S_{x}$ remains constant [Figs. 5(c) and 5(d)], so these modes are fully compensated. And for $k_{y}$ close to points 1 and 2 [Figs. 5(e) and 5(f)], the average value of $S_{x}$ increases, which means that modes are amplified. Such discontinuities in isofrequency contours, followed by the change of the sign of $\operatorname{Im}\left(k_{x}\right)$, mean that the system becomes unstable for $\varepsilon_{2}^{\prime \prime} \gtrsim 0.02$. Therefore, at this frequency stable loss compensation can be obtained only for a certain value of $k_{y}$.

When the normalized frequency is $k_{0} D=0.59$ [Figs. $4(\mathrm{~d})$ 4(f)] or $k_{0} D=0.75$ [Figs. 4(g)-4(i)], one can see that in the presence of a certain amount of gain $\left(\varepsilon_{2}^{\prime \prime} \approx 0.06\right.$ for $k_{0} D=$ 

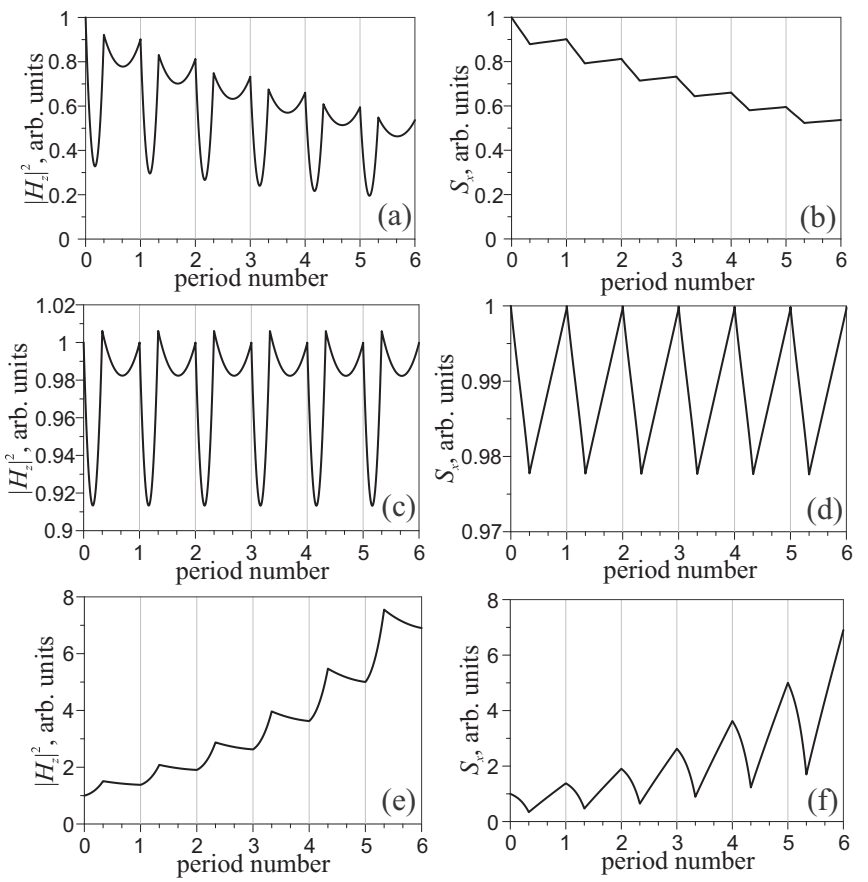

FIG. 5. (a), (c), (e) Magnetic field distribution $\left|H_{z}(x)\right|^{2}$ and (b), (d), (f) $x$ component of Poynting vector $S_{x}(x)$. The plots correspond to the dispersion points marked in Fig. 4(b): (a), (b) 5, 6, (c), (d) 3, 4, and (e), (f) $1,2$.

0.59 and $\varepsilon_{2}^{\prime \prime} \approx 0.03$ for $k_{0} D=0.75$ ), the imaginary part of the $x$ component of the Bloch wave vector almost vanishes at all possible values of $k_{y}$. At these frequencies, with an addition of the right amount of gain, the metamaterial becomes lossless for every angle of incidence.

In the canalization regime [see Figs. 4(j) and 4(k)], an addition of a small amount of gain $\left(\varepsilon_{2}^{\prime \prime}=0.013\right)$ allows one to compensate losses for a certain range of $k_{y}$, where the isofrequency curves are close to straight lines, responding to canalization regime. Further increase of gain leads to the appearance of modes with negative imaginary part of $k_{x}$, turning the system into an unstable state.

\section{B. Longitudinal propagation}

This regime describes the modes which would be excited if the structure was finite in the $y$ direction [see Figs. 4(j) and $4(\mathrm{k})$ ], in the so-called end-fire excitation. In this case, $k_{x}$ is real, and there exist an infinite number of complex modes for any given $k_{x}$, because the system is periodic. Here we take into account only the modes with the smallest imaginary parts of $k_{y}$, i.e., those modes which have the largest propagation distances. For both $k_{0} D=0.3$ and $k_{0} D=0.65$, the hyperbolic dispersion branches have strong dependence on $k_{x}$. For $k_{0} D=0.3$, there exists only a hyperbolic branch, and it has a minimum attenuation at $k_{x}=0$ [dashed line in Fig. 6(d)]. With an increase of frequency, at $k_{0} D \approx 0.58$ (close to the bulk plasmon frequency), an elliptical branch appears, and at $k_{x} \approx 0$ its imaginary part is much smaller than the imaginary part of the hyperbolic branch, while the hyperbolic branch now has minimum losses at the edges of the Brillouin zone $k_{x}= \pm 1$ [dashed line in Fig. 6(b)]. So, in the case of
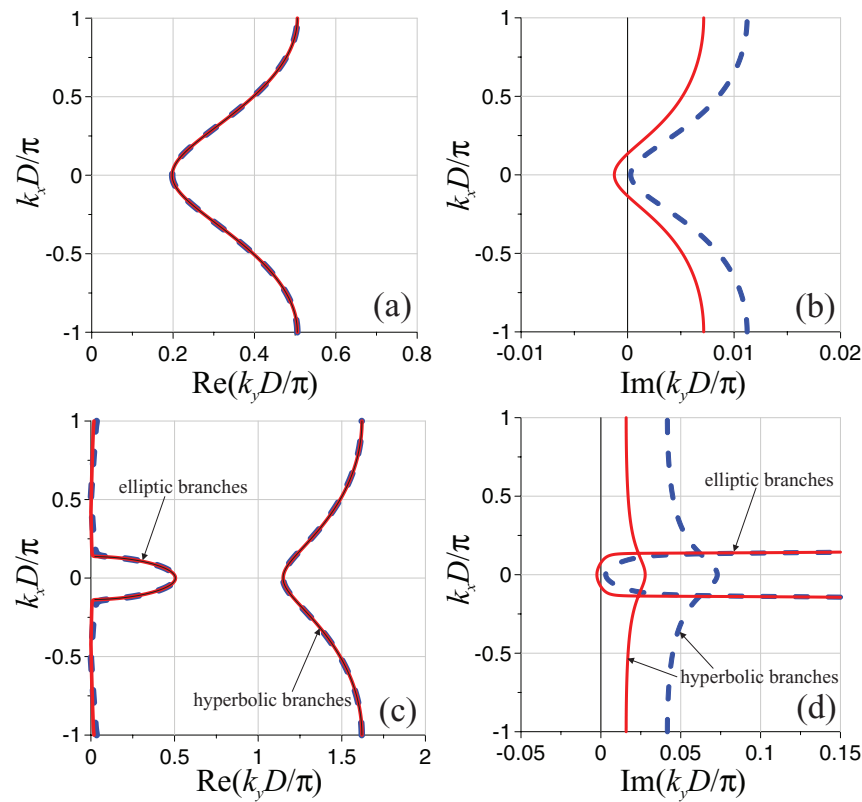

FIG. 6. (Color online) Examples of the isofrequency contours $k_{y}\left(k_{x}\right)$ for the longitudinal propagation (complex-valued $k_{y}$ and realvalued $k_{x}$ ) for different frequencies: (a), (b) $k_{0} D=0.3$; (c), (d) $k_{0} D=$ 0.65 . Thin solid curves correspond to the case of zero losses, dashed curves to the case with losses, and thick solid curves to the case with losses and gain.

the longitudinal propagation, losses can be compensated only at the center of the Brillouin zone for the hyperbolic branch below the bulk plasmon frequency, and for the elliptical branch above the bulk plasmon frequency.

\section{CONCLUSIONS}

We have analyzed the properties of MDLM with gain and different regimes of loss compensation in these structures. We have shown that in the case of the transverse propagation at low frequencies, the structure is sensitive to gain at a certain angle of incidence. As a result, only for a particular angle the loss can be fully compensated by gain avoiding convective instability, whereas for other angles the wave attenuation is weakly affected. Close to the ENZ frequency and SPP resonance frequency, the value of gain required for the complete compensation of losses weakly depends on the angle on incidence; therefore, at these frequencies almost full compensation is possible. In the canalization regime, loss compensation can be achieved for a wider spatial spectrum, due to a weak dependence of the attenuation rate on the longitudinal component of the wave vector.

For the longitudinal propagation, losses of the hyperbolic branch can be compensated at the center of the Brillouin zone below the bulk plasmon frequency. For higher frequencies, an elliptic branch appears for which the losses can be compensated, whereas losses of the hyperbolic branch are too severe, and the corresponding modes remain strongly suppressed by losses.

As a next step, it would be important to consider nonlinear effects of gain saturation and quantum electrodynamics effects, such as Purcell effect and nonradiative decay rate change, 
which can affect negatively the performance of gain media for loss compensation. ${ }^{19,29,44}$ It was shown ${ }^{29}$ that ultrahigh values of the Purcell factor are inherent to metal-dielectric structures due to a high density of states with hyperbolic isofrequency contours. This can increase the requirements for loss compensation, as was shown for plasmonic waveguides. ${ }^{19}$ Besides that, from a practical point of view, it will be also important to study the temporal dynamics of pulse propagation $^{23,45}$ in such loss compensated structures.

\section{ACKNOWLEDGMENTS}

This work has been supported by the Ministry of Education and Science of the Russian Federation, Projects No. 11.G34.31.0020, No. 14.B37.21.0907, No. 14.B37.21.1283, and No. 14.B37.21.1649, by RFBR Projects No. 12-02-00853a and No. 10-02-01016-a, by the Dynasty Foundation, Grant of President of Russian Federation MD-6805.2013.2, and the Australian Research Council.

\section{APPENDIX: PERTURBATION THEORY} form:

Dispersion equation (1) can be written in the following

$$
\begin{aligned}
& F\left(k_{x}, k_{y}, \varepsilon_{1}, \varepsilon_{2}\right) \\
& \equiv\left[\cos \left(k_{x} D\right)-\cos \left(q_{1} d_{1}\right) \cos \left(q_{2} d_{2}\right)\right. \\
& \left.\quad+\frac{1}{2}\left(\frac{\varepsilon_{2} q_{1}}{\varepsilon_{1} q_{2}}+\frac{\varepsilon_{1} q_{2}}{\varepsilon_{2} q_{1}}\right) \sin \left(q_{1} d_{1}\right) \sin \left(q_{2} d_{2}\right)\right]=0,
\end{aligned}
$$

where $\varepsilon_{1}, \varepsilon_{2}$ are the real-valued parameters in the case of zero losses and gain $\left(\gamma=0, \varepsilon_{2}^{\prime \prime}=0\right)$.

When we add a small imaginary part to $\varepsilon_{1}$ and to $\varepsilon_{2}$, we can write in the "first-order" approximation:

$$
\partial_{k_{x}} F \Delta k_{x}+\partial_{k_{y}} F \Delta k_{y}+\partial_{\varepsilon_{1}} F\left(i \Delta \varepsilon_{1}\right)+\partial_{\varepsilon_{2}} F\left(i \Delta \varepsilon_{2}\right)=0,
$$

where $\partial_{j} F$ are the partial derivatives of the function $F$, $\Delta \varepsilon_{1}, \Delta \varepsilon_{2}$ are the shifts of the imaginary parts of permittivities, and $\Delta k_{x}, \Delta k_{y}$ are small additions to the wave vector components. Here we neglect a small change in the real part of $\varepsilon_{1}$ caused by a nonzero value of $\gamma$. In the case of transverse propagation we have $\Delta k_{y}=0$, therefore

$$
\Delta k_{x} D=i \frac{\partial_{\varepsilon_{1}} F \Delta \varepsilon_{1}+\partial_{\varepsilon_{2}} F \Delta \varepsilon_{2}}{\sin \left(k_{x} D\right)} .
$$

In terms of the perturbation theory, the condition of the exact compensation is

$$
\partial_{\varepsilon_{1}} F \Delta \varepsilon_{1}+\partial_{\varepsilon_{2}} F \Delta \varepsilon_{2}=0
$$

For $k_{y}=0, k_{x}$ has some finite real value in the allowed band (for $k_{0} D \gtrsim 0.58$ ); thereby, according to Eq. (A1), $\Delta k_{x}$ has some finite imaginary value. Then, by fulfilling the condition (A2), exact loss compensation can be achieved in this case.

In the band gaps, $k_{x}$ is an imaginary number, so $\Delta k_{x}$ is real valued and $k_{x} D / \pi$ has a change only in the real part, as one can see in Fig. 4. Also, in Fig. 4(d) there are very small band gaps at around $k_{y}= \pm 0.44$. And one can see in Figs. 4(e) and 4(f) that losses are relatively small (comparing to losses at other values of $k_{y}$ ) at these points, and gain slightly affects the value of $\operatorname{Im}\left(k_{x} D / \pi\right)$.

When $\partial_{k_{y}} k_{x} \rightarrow \infty$, in Figs. 4(a), 4(d), 4(g), and 4(j) isofrequency contours are close to the straight vertical lines, which occur at the edges of the allowed bands, when $k_{x} D / \pi \rightarrow 0$ or \pm 1 (and therefore $\partial_{k_{x}} F \rightarrow 0$ ). In this case, $\Delta \varepsilon_{1}$ and $\Delta \varepsilon_{2}$, i.e., losses and gain, strongly affect the imaginary part of the $x$ component of the Bloch wave vector. Any small deviation from the condition (A2) provides large effective losses or gain, so exact compensation becomes practically impossible. It happens because $\partial_{k_{x}} F \rightarrow 0$ corresponds to the "slow light" regime, and in this case effective losses and gain increase proportionally to the light slowdown. ${ }^{46}$
${ }^{1}$ R. Wangberg, J. Elser, E. E. Narimanov, and V. A. Podolskiy, J. Opt. Soc. Am. B 23, 498 (2006).

${ }^{2}$ J. B. Pendry, Phys. Rev. Lett. 85, 3966 (2000).

${ }^{3}$ P. A. Belov and Y. Hao, Phys. Rev. B 73, 113110 (2006).

${ }^{4}$ X. Li, S. He, and Y. Jin, Phys. Rev. B 75, 045103 (2007).

${ }^{5}$ Z. Liu, H. Lee, Y. Xiong, C. Sun, and X. Zhang, Science 315, 1686 (2007).

${ }^{6}$ W. Cai, U. K. Chettiar, A. V. Kildishev, and V. M. Shalaev, Opt. Express 16, 5444 (2008).

${ }^{7}$ Y. Xiong, Z. Liu, and X. Zhang, Appl. Phys. Lett. 93, 111116 (2008).

${ }^{8}$ S. Xiao, V. P. Drachev, A. V. Kildishev, X. Ni, U. K. Chettiar, H.-K. Yuan, and V. M. Shalaev, Nature (London) 466, 735 (2010).

${ }^{9}$ C. M. Soukoulis and M. Wegener, Science 330, 1633 (2010).

${ }^{10}$ M. I. Stockman, Phys. Rev. Lett. 106, 156802 (2011).

${ }^{11}$ J. Grgić, J. R. Ott, F. Wang, O. Sigmund, A.-P. Jauho, J. Mørk, and N. A. Mortensen, Phys. Rev. Lett. 108, 183903 (2012).

${ }^{12}$ N. N. Rozanov, S. V. Fedorov, R. S. Savelev, A. A. Sukhorukov, and Y. S. Kivshar, JETP 114, 782 (2012).
${ }^{13}$ A. Fang, T. Koschny, and C. M. Soukoulis, Phys. Rev. B 82, 121102 (2010).

${ }^{14}$ A. Fang, Z. Huang, T. Koschny, and C. M. Soukoulis, Photon. Nanostruct. 10, 276 (2012).

${ }^{15}$ J. M. Hamm, S. Wuestner, K. L. Tsakmakidis, and O. Hess, Phys. Rev. Lett. 107, 167405 (2011).

${ }^{16}$ M. A. Noginov, G. Zhu, M. Bahoura, J. Adegoke, C. E. Small, B. A. Ritzo, V. P. Drachev, and V. M. Shalaev, Opt. Lett. 31, 3022 (2006).

${ }^{17}$ M. A. Noginov, V. A. Podolskiy, G. Zhu, M. Mayy, M. Bahoura, J. A. Adegoke, B. A. Ritzo, and K. Reynolds, Opt. Express 16, 1385 (2008).

${ }^{18}$ I. D. Leon and P. Berini, Nat. Photon. 4, 382 (2010).

${ }^{19}$ J. B. Khurgin and G. Sun, Appl. Phys. Lett. 100, 011105 (2012).

${ }^{20}$ S. A. Ramakrishna and J. B. Pendry, Phys. Rev. B 67, 201101 (2003).

${ }^{21}$ M. A. Vincenti, D. de Ceglia, V. Rondinone, A. Ladisa, A. D’Orazio, M. J. Bloemer, and M. Scalora, Phys. Rev. A 80, 053807 (2009).

${ }^{22}$ X. Ni, S. Ishii, M. D. Thoreson, V. M. Shalaev, S. Han, S. Lee, and A. V. Kildishev, Opt. Express 19, 25242 (2011). 
${ }^{23}$ A. Fang, T. Koschny, M. Wegener, and C. M. Soukoulis, Phys. Rev. B 79, 241104 (2009).

${ }^{24}$ N. Meinzer, M. Ruther, S. Linden, C. M. Soukoulis, G. Khitrova, J. Hendrickson, J. D. Olitzky, H. M. Gibbs, and M. Wegener, Opt. Express 18, 20535 (2010).

${ }^{25}$ K. Tanaka, E. Plum, J. Y. Ou, T. Uchino, and N. I. Zheludev, Phys. Rev. Lett. 105, 227403 (2010).

${ }^{26}$ A. Fang, Z. Huang, T. Koshny, and C. M. Soukoulis, Opt. Express 19, 12688 (2011).

${ }^{27}$ R. Marani, A. D’Orazio, V. Petruzzelli, S. G. Rodrigo, L. MartínMoreno, F. J. García-Vidal, and J. Bravo-Abad, New J. Phys. 14, 013020 (2012).

${ }^{28}$ A. P. Vinogradov, E. S. Andrianov, A. A. Puhov, A. V. Dorofeenko, and A. A. Lisyansky, Phys. Usp. 55, 1046 (2012).

${ }^{29}$ I. Iorsh, A. Poddubny, A. Orlov, P. Belov, and Y. S. Kivshar, Phys. Lett. A 376, 185 (2012).

${ }^{30}$ H. N. Krishnamoorthy, Z. Jacob, E. Narimanov, I. Kretzschmar, and V. M. Menon, Science 336, 205 (2012).

${ }^{31}$ J. Elser, V. A. Podolksiy, I. Salakhutdinov, and I. Avrutsky, Appl. Phys. Lett. 90, 191109 (2007).

${ }^{32}$ A. P. Vinogradov, A. V. Dorofeenko, and I. A. Nechpurenko, Metamaterials 4, 181 (2010).

${ }^{33}$ A. A. Orlov, P. M. Voroshilov, P. A. Belov, and Y. S. Kivshar, Phys. Rev. B 84, 045424 (2011).
${ }^{34}$ V. P. Drachev, U. K. Chettiar, A. V. Kildishev, H.-K. Yuan, W. Cai, and V. M. Shalaev, Opt. Express 16, 1186 (2008).

${ }^{35}$ P. B. Johnson and R. W. Christy, Phys. Rev. B 6, 4370 (1972).

${ }^{36}$ L. A. Coldren and S. W. Corzine, Diode Lasers and Photonic Integrated Circuits (John Wiley \& Sons, New York, 1995).

${ }^{37}$ S. M. Rytov, Sov. Phys. JETP 2, 466 (1956).

${ }^{38}$ A. Orlov, I. Iorsh, P. Belov, and Y. Kivshar, Opt. Express 21, 1593 (2013).

${ }^{39}$ M. Premaratne and G. P. Agrawal, Light Propagation in Gain Media: Optical Amplifiers (Cambridge University Press, Cambridge, UK, 2011).

${ }^{40}$ R. V. Karapetyan, Laser Phys. 17, 1053 (2007).

${ }^{41}$ A. V. Dorofeenko, A. A. Zyablovsky, A. A. Pukhov, A. A. Lisyansky, and A. P. Vinogradov, Phys. Usp. 55, 1080 (2012).

${ }^{42}$ W. Koechner and M. Bass, Solid State Lasers (Springer, New York, 2003).

${ }^{43}$ P. M. Bolger, W. Dickson, A. V. Krasavin, L. Liebscher, S. G. Hickey, D. V. Skryabin, and A. V. Zayats, Opt. Lett. 35, 1197 (2010).

${ }^{44}$ J. Kim, V. P. Drachev, Z. Jacob, G. V. Naik, A. Boltasseva, E. E. Narimanov, and V. M. Shalaev, Opt. Express 20, 8100 (2012).

${ }^{45}$ Z. Huang, T. Koschny, and C. M. Soukoulis, Phys. Rev. Lett. 108, 187402 (2012).

${ }^{46}$ T. Baba, Nat. Photon. 2, 465 (2008). 